\title{
Bilateral Total Knee Arthroplasty for Charcot Arthropathy After Cauda Equine Syndrome: A Case Report
}

\author{
Athanasios Karageorgos ${ }^{\mathrm{a}, \mathrm{d}}$, Andreas X. Papadopoulos ${ }^{\mathrm{a}}$, \\ Ioannis D. Gelalis ${ }^{\mathrm{b}}$, Charalampos Matzaroglou ${ }^{\mathrm{c}}$
}

\begin{abstract}
Charcot arthropathy of the knee is a relatively rare and poorly understood condition. Diagnosis requires detailed history of the patient, radiological investigation and exclusion of other causes of arthropathy. Conservative treatment is sufficient only in early stages. In late stages, either arthrodesis or total knee arthroplasty is the treatment of choice. We report a case of a 65 -year-old woman who presented with Charcot arthropathy in both knees, after a spinal fracture 35 years ago, which caused cauda equine syndrome with diminished sensation of both legs. She underwent bilateral total knee arthroplasty using hinged knee prosthesis.
\end{abstract}

Keywords: Charcot knee; Neuropathic knee arthropathy; Cauda equine

\section{Introduction}

Charcot arthropathy is a relatively rare and poorly understood condition. It is a progressive degenerative disease of the joint that may lead to severe deformity and dysfunction. Bilateral knee neuropathic arthropathy is an exceedingly rare condition [1]. Given the rarity of Charcot arthropathy of the knee in general treatment is still controversial, with the trend nowadays toward total knee arthroplasty (TKA) [2].

Pathomechanism of the disease is still unknown. However, it is believed that the diminution or absence of nociception, results in poor joint protection and undetected microtrauma may lead to development of bone destruction and attenuation of ligaments [3]. Furthermore, according to neurovascular the-

Manuscript submitted December 26, 2019, accepted January 13, 2020

${ }^{a}$ Department of Orthopaedic Surgery, Olympion Medical Center, Patras, Greece

bOrthopaedic Department, University of Ioannina, Greece

'Orthopaedic Surgery and Traumatology Department of Rehabilitation Sciences, University of Patras, Greece

${ }^{\mathrm{d} C o r r e s p o n d i n g ~ A u t h o r: ~ A t h a n a s i o s ~ K a r a g e o r g o s, ~ D e p a r t m e n t ~ o f ~ O r t h o p a e d i c ~}$ Surgery, Olympion Medical Center, Patras, Greece.

Email: a.karageorgos@hotmail.com

doi: https://doi.org/10.14740/jmc3412 ory, neurally stimulated vascular reflexes may provoke bone resorption. In the first half of the 20th century syphilis was the main cause of Charcot arthropathy. As syphilis declined due to antibiotic evolution, diabetes mellitus became the primary etiology [4-6].

Diagnosis of Charcot arthropathy should include detailed history taking, and a complete physical examination, including neurologic tests. It is based on clinical findings such as warm and swollen painless joint and later radiographic evaluation, which may reveal joint dislocation, fractures, or deformities [7].

We report a case of Charcot arthropathy affecting both knees after a fifth lumbar vertebral (O5) fracture 35 years ago, which led to cauda equine syndrome and treated with bilateral total knee arthroplasty.

\section{Case Report}

A 65-year-old woman presented to the orthopaedic office on a wheelchair. The patient had suffered an O5 fracture 35 years ago which was treated conservatively, and produced a cauda equine syndrome. The symptoms of urinary incontinence, diminished sensation in both legs distally to the middle of both thighs and deep tendon reflexes were remaining. Furthermore, she presented with muscular weakness $(2+/ 5)$ of both feet, and she used feet orthoses. The quadriceps strength was uneventful. The patient had no notable medical history concerning diabetes mellitus, syphilis, lacunar infarcts, rheumatologic disease and osteoarthritis, and she denied tobacco and alcohol use. She had suffered a right middle tibia fracture 5 years ago, which was treated with intramedullary nailing and fused uneventfully. After a period of 2 months she regained her prior walking ability and was able to walk using a walker. The last 2 years she gradually lost her walking ability due to gross instability of both knees.

The main complaints were progressively swollen knees with mild pain and increasingly instability with valgus deformity and crepitus for the last 2 years. Since then the patient was unable to weight bear due to gross instability, and she used a wheelchair. During her admission to the orthopaedic office both knees had similar clinical deterioration and radiological abnormalities. Gross abnormal mobility was present on clinical examination in both sagittal and coronal planes. Anterior and posterior drawer test were positive. Varus and valgus instability test were also positive. 


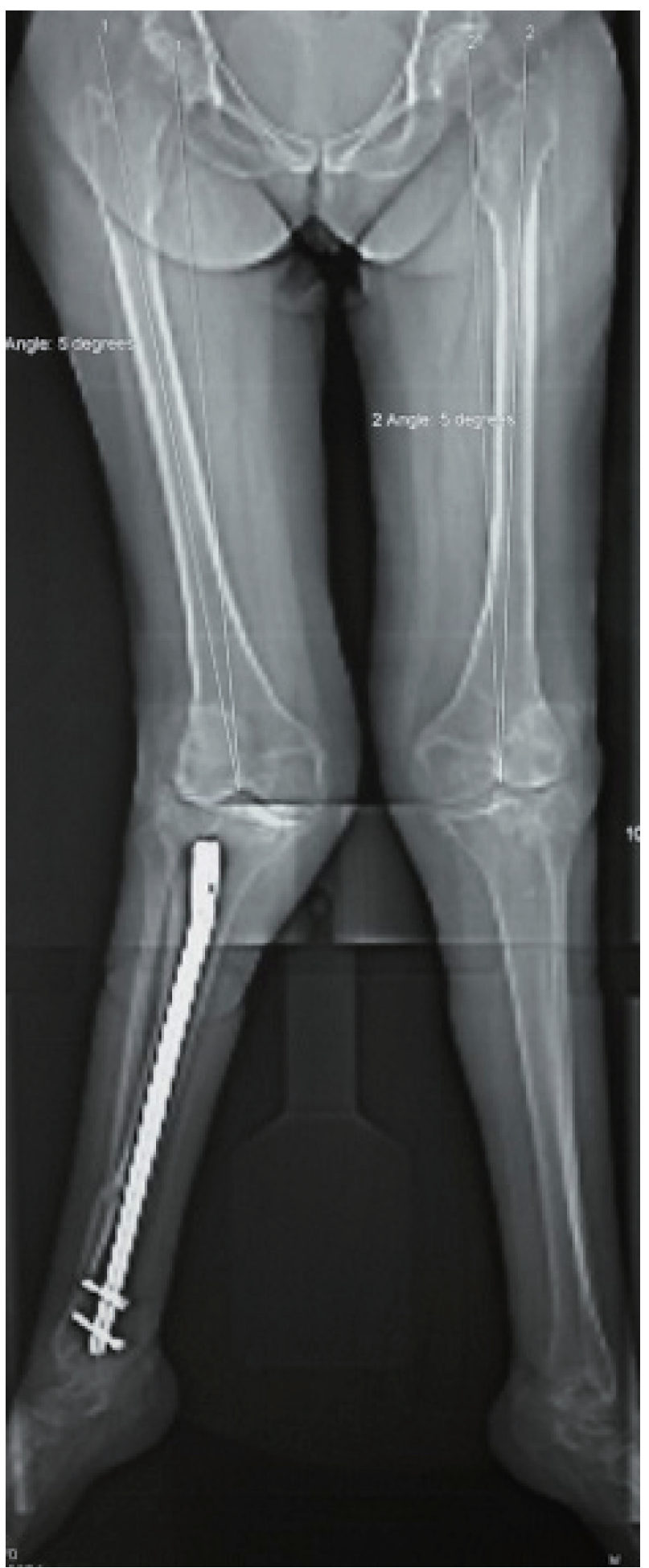

Figure 1. Preoperative face $\mathrm{X}$-ray, before nail removal showing excessive valgus deformity.

The diagnosis of Charcot arthropathy was established using knee X-rays, which showed marked destructive changes, sclerosis, dislocation, and subchondral fractures of the knee joints (Figs. 1, 2).

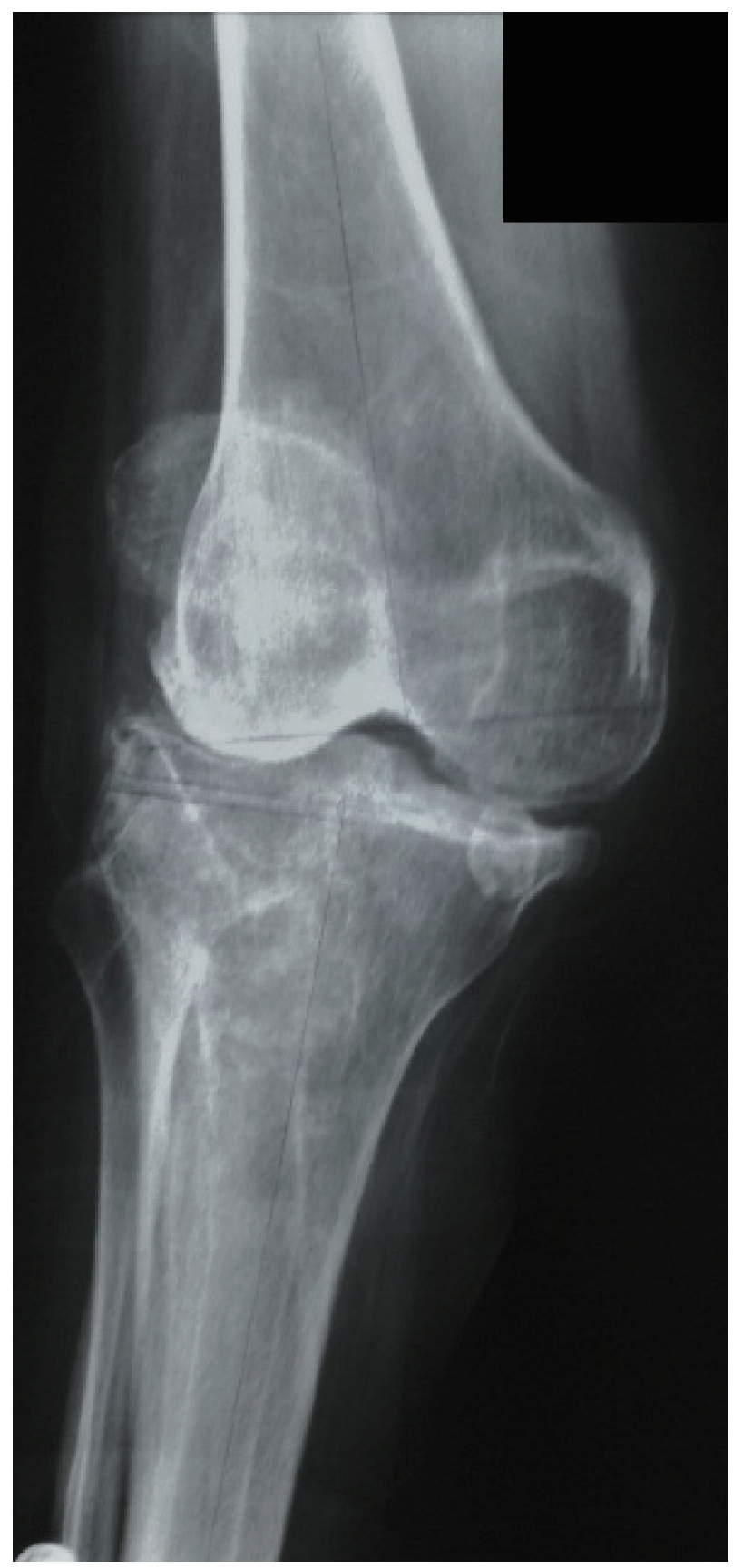

Figure 2. Right knee face X-ray after nail removal. Destructive changes are apparent.

Due to the progressive inability to ambulate the patient decided to be treated with total knee arthroplasty (TKA). She underwent removal of the intramedullary nail first. Two months later she was operated on her right knee using cemented rotating hinged knee prosthesis, in order to accommodate bone loss and ligamentous insufficiency. On postoperative day 2, the patient could partially weight bear. Wound healing was uneventful, and on postoperative day 20 knee range of motion (ROM) was $115^{\circ} / 0^{\circ} 0^{\circ}$ (flexion/extension/hyperextension). Knee stability was restored, and she was allowed to weight bear. Three months 


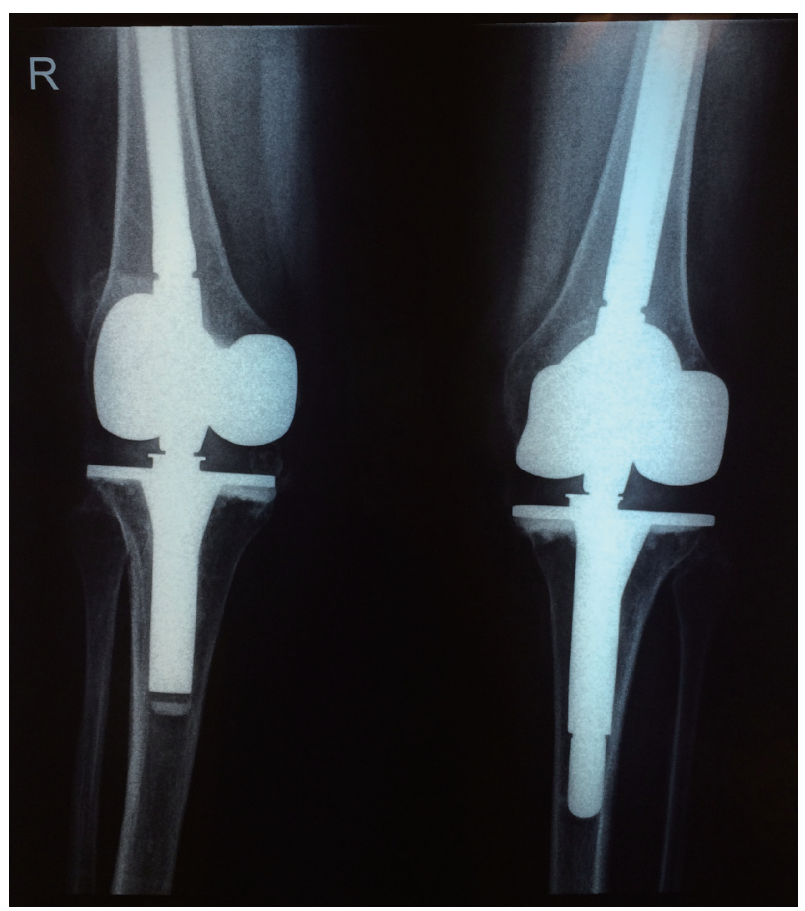

Figure 3. Twelve years postoperative $\mathrm{X}$-ray, showing good component alignment, without loosening.

later the patient underwent TKA at her left knee using identical technique. Postoperative course was uncomplicated, and on day 20 , ROM was $120^{\circ} / 0^{\circ} / 0^{\circ}$. Six weeks later she was able to walk with the aid of a walker and was satisfied with the result.

We assessed the clinical outcome using the Knee Society Score (KSS) [8], which was calculated preoperatively and at the final evaluation 12 years postoperatively. There was an improvement from 25 to 90 points in knee score and from 0 to 30 points in function score, although she was continued to use walker due to her muscular weakness of both feet. Radiological evaluation also took place at the final follow-up, using radiographs. The position of components was evaluated for proper placement and loosening, with no apparent sings of malposition and progressive loose lines (Fig. 3).

\section{Discussion}

Neuropathic arthropathy was described by Jean Martin Charcot in 1868 as the progressive destruction of bone and soft tissue in patients with peripheral neuropathy. Almost 100 years later, Eichenholtz stated that the knee was the most common site of neuroarthropathy, and all these patients had tabes dorsalis. Since then and due to antibiotic evolution, the incidence of neurosyphilis has been declining, with diabetes mellitus constituting the most common reason for neuroarthropathy of joints in the lower extremity [9]. Diabetic neuropathy most commonly involves the tarsal, tarsometatarsal and ankle joints [10]. Other causes are leprosy, meningomyelocele, syringomyelia and spinal cord injury $[11,12]$. Our case is the only one in the literature that followed vertebral fracture and cauda equine syndrome.

The pathophysiology of Charcot arthropathy is not fully understood. The current accepted theory states that in susceptible individuals with peripheral neuropathy, an unregulated inflammatory process is triggered which leads to an increase expression of the polypeptide receptor activator of nuclear factor kappa-b ligand (RANKL). RANKL triggers the synthesis of nuclear transcription factor, nuclear factor- $\kappa \beta(\mathrm{NF}-\kappa \beta)$, and this in terms stimulates the maturation of osteoclasts from osteoclast precursor cells. Furthermore NF- $\kappa \beta$ stimulates the production of the glycopeptide osteoprotegerin (OPG) from osteoblasts. All these events lead to continuing local osteolysis. The damaged nervous endings may also decrease the secretion of the calcitonin gene-related peptide (CGRP). This peptide works as an antagonist of the RANKL synthesis and at the same time is responsible for the normal integrity of the joint capsule [13].

The diagnosis is based on patient's history, clinical examination, and imaging methods. Risk factors such as diabetes mellitus, syphilis, leprosy, alcoholism, syringomyelia, lacunar infarcts etc., which can lead to loss of deep sensation and proprioception, should be identified.

Typical symptoms in clinical examination of active neuropathic arthropathy are edema, erythema, warmth, and more than $2{ }^{\circ} \mathrm{C}$ difference in local temperature in comparison to the contralateral extremity. Pain occurs in about $50 \%$ of neuropathy cases [14]. Furthermore, the degree of pain does not correlate with the degree of joint destruction [7]. Differential diagnosis in acute stage of disease should be done especially between septic arthritis, osteomyelitis and microcrystalline arthritis. Our case presented with mild pain due to loss of sensitivity, despite the gross instability of both knees.

Radiograph is the preferred examination method concerning the imaging methods. The Eichenholtz classification [15] is still in use and it consists of three stages. Stage of development, which is an early stage of the disease, shows evidence of debris formation, fragmentation, disruption and dislocation of the articular margins. Stage of coalescence is characterized by absorption of much or all of the fine debris, sclerosis, and fusion of most large fragments. Finally, stage of reconstruction presents decrease sclerosis, rounding of major fragments, and some kind of reformation of joint architecture. Recently magnetic resonance imaging (MRI) has been broadly used to detect this condition early, as changes on X-ray are typically delayed and have low sensitivity [16]. Bone marrow edema as a finding in a patient with risk factors may depict neuropathic arthropathy in early stages.

The treatment of neuropathic arthropathy is conservative at the early stages of the disease prior to radiographic abnormalities. It is based on immobilization and complete absence of weight bearing for the affected extremity in the active stage. To support healing bisphosphonates, intranasal calcitonin [17] and teriparatide [18] have been used, but their efficacy is yet to be fully demonstrated. When the knee joint is affected, and radiological abnormalities are apparent, conservative treatment is insufficient [19]. Arthrodesis has been the mainstay of operative treatment in the late stages of neuroarthropathy, despite a high incidence of failure [20]. Knee arthroplasty has been considered to be an absolute contraindication because 
of the high incidence of serious complications [21]. However in recent years some authors have shown satisfactory results after knee arthroplasty in patients with neuropathic arthropathy $[21,22]$. High incidence of serious complications, such as periprosthetic infection, aseptic loosening, and periprosthetic fracture, in these patients are related to significant bone loss, poor bone quality and ligamentous laxity. Recommendations for successful total knee arthroplasty include bone grafting or custom-augmented prosthesis to repair bony defects, correct ligamentous balancing, total synovectomy, and a complete armamentarium of the new implants. Furthermore, a better outcome is obtained if implantation occurs after the initial developmental phase [23].

\section{Conclusions}

Late stage neuropathic arthropathy of the knee causes significant functional deficits, and its treatment is very challenging. There is still a lack of consensus regarding the optimal treatment when operative management is indicated. Although knee arthroplasty recently provides promising results, it is associated with high complication rates, is technically demanding, and needs a complete armamentarium of the new implants.

\section{Acknowledgments}

None to declare.

\section{Conflict of Interest}

None to declare.

\section{Financial Disclosure}

None to declare.

\section{Informed Consent}

Informed consent has been received.

\section{Author Contributions}

Athanasios Karageorgos: substantial contribution of the conception and design of the work; Andreas X. Papadopoulos: acquisition and analysis of data for the work; Gelalis D. Ioannis: drafting the work; Charalampos Matzaroglou: final approval of the version to be published.

\section{Data Availability}

The authors declare that data supporting the findings of this study are available within the article.

\section{References}

1. Fullerton BD, Browngoehl LA. Total knee arthroplasty in a patient with bilateral Charcot knees. Arch Phys Med Rehabil. 1997;78(7):780-782.

2. Zeng M, Xie J, Hu Y. Total knee arthroplasty in patients with Charcot joints. Knee Surg Sports Traumatol Arthrosc. 2016;24(8):2672-2677.

3. Sequeira W. The neuropathic joint. Clin Exp Rheumatol. 1994;12(3):325-337.

4. Cassidy RC, Shaffer WO. Charcot arthropathy because of congenital insensitivity to pain in an adult. Spine J. 2008;8(4):691-695.

5. Feldman DS, Ruchelsman DE, Spencer DB, Straight JJ, Schweitzer ME, Axelrod FB. Peripheral arthropathy in hereditary sensory and autonomic neuropathy types III and IV. J Pediatr Orthop. 2009;29(1):91-97.

6. Sudanese A, Paderni S, Guerra E, Bertoni F. Neurogenic arthropathy of the knee due to chronic alcoholism: two case reports. Chir Organi Mov. 2003;88(4):427-434.

7. Babazadeh S, Stoney JD, Lim K, Choong PF. Arthroplasty of a Charcot knee. Orthop Rev (Pavia). 2010;2(2):e17.

8. Insall JN, Dorr LD, Scott RD, Scott WN. Rationale of the Knee Society clinical rating system. Clin Orthop Relat Res. 1989;248:13-14.

9. Rajbhandari SM, Jenkins RC, Davies C, Tesfaye S. Charcot neuroarthropathy in diabetes mellitus. Diabetologia. 2002;45(8):1085-1096.

10. Gupta R. A short history of neuropathic arthropathy. Clin Orthop Relat Res. 1993;296:43-49.

11. Jones EA, Manaster BJ, May DA, Disler DG. Neuropathic osteoarthropathy: diagnostic dilemmas and differential diagnosis. Radiographics. 2000;20(suppl_1):S279-293.

12. Viens NA, Watters TS, Vinson EN, Brigman BE. Case report: Neuropathic arthropathy of the hip as a sequela of undiagnosed tertiary syphilis. Clin Orthop Relat Res. 2010;468(11):3126-3131

13. Rogers LC, Frykberg RG, Armstrong DG, Boulton AJ, Edmonds M, Van GH, Hartemann A, et al. The Charcot foot in diabetes. Diabetes Care. 2011;34(9):2123-2129.

14. Botek G, Anderson MA, Taylor R. Charcot neuroarthropathy: An often overlooked complication of diabetes. Cleve Clin J Med. 2010;77(9):593-599.

15. Eichenholtz SN. Charcot joints. With a foreword by P.D. Wilson. Springfield (III): Charles C. Thomas. 1966

16. Morrison WB, Ledermann HP. Work-up of the diabetic foot. Radiol Clin North Am. 2002;40(5):1171-1192.

17. Bem R, Jirkovska A, Fejfarova V, Skibova J, Jude EB. Intranasal calcitonin in the treatment of acute Charcot neuroosteoarthropathy: a randomized controlled trial. Diabetes Care. 2006;29(6):1392-1394.

18. Tamai K, Takamatsu K, Kazuki K. Successful treatment of nonunion with teriparatide after failed ankle arthrodesis for Charcot arthropathy. Osteoporos Int. 2013;24(10):2729-2732.

19. Nielson DL, Armstrong DG. The natural history of 
Charcot's neuroarthropathy. Clin Podiatr Med Surg. 2008;25(1):53-62, vi.

20. Drennan DB, Fahey JJ, Maylahn DJ. Important factors in achieving arthrodesis of the Charcot knee. J Bone Joint Surg Am. 1971;53(6):1180-1193.

21. Parvizi J, Marrs J, Morrey BF. Total knee arthroplasty for neuropathic (Charcot) joints. Clin Orthop Relat Res.
2003;416:145-150.

22. Bae DK, Song SJ, Yoon KH, Noh JH. Long-term outcome of total knee arthroplasty in Charcot joint: a 10- to 22year follow-up. J Arthroplasty. 2009;24(8):1152-1156.

23. Yoshino S, Fujimori J, Kajino A, Kiowa M, Uchida S. Total knee arthroplasty in Charcot's joint. J Arthroplasty. 1993;8(3):335-340. 